\title{
Il project financing per i beni culturali italiani
}

\author{
di Osvaldo Maria Baione, Francesca Grotti e Emanuele Teti
}

Partendo dall'evidenza del ruolo delle forme di finanza innovativa nelle iniziative pubbliche, obiettivo del lavoro sarà indagare sull'applicazione di tale strumenti a quanto forse di più "pubblico" vi è in Italia: la nostra Cultura. Considerate le condizioni di necessità in cui riversa il settore culturale italiano, le Partnership Pubblico-Privato rappresentano interessanti alternative per la Pubblica Amministrazione competente nell'ottica di adempiere ai suoi doveri costituzionali. Cresce la consapevolezza dell'insufficienza della sola finanza statale tradizionale, e con essa il numero dei sostenitori dell'implementazione, anche in cultura, di forme alternative di finanziamento e management.

Il Project Financing si inserisce in questo quadro come modalità di finanziamento innovativa e adeguata, ereditata dal sistema inglese e ad oggi utilizzata principalmente per la realizzazione di grandi infrastrutture o riqualificazioni di interesse pubblico. Introdotto nella regolamentazione nel 1998 e successivamente rivisitato, tale strumento è tuttavia stato impiegato efficacemente in un progetto culturale solo nel 2013, in occasione della riapertura del Corpo Centrale di Villa Reale di Monza.

Questo lavoro analizza i pro e contro dell'applicazione della finanza di progetto alla cultura, traendo spunto da questo primo caso di riferimento.

Parole chiave: project finance, finanza, cultura, beni culturali, finanziamenti.

Beginning with the importance of innovative financial methods within public initiatives, the purpose of this work is to further examine their application to the most "public" thing in Italy: our Culture. Considering the difficulties of the Italian public sector, Private-Public Partnerships represent interesting alternatives to the Public Administration in charge to fulfil its constitutional duties. The awareness of traditional finance inadequacy and insufficiency increases along with the number of supporters of alternative forms of management and financing, in culture too.

Project financing (PF) belongs to this group as a new and suitable method, inherited from the English system and nowadays applied mostly for the realization or requalification of public infrastructures. 
Although it was introduced in the Italian regulation in 1998 and then modified up until the latest version of 2008, this tool had been used efficiently in a cultural project in 2013 only: the requalification of the Central body of Villa Reale, in Monza. This case will be the practical evidence and reference in order to grasp most relevant pros and cons in cultural project financing experiences.

Keywords: project finance, finance, culture, cultural heritage, financing

JEL Classification: G2, G2, E6.

\section{Premessa}

Il capostipite per eccellenza tra gli strumenti utilizzabili dagli operatori privati per finanziare un'operazione di Partnership Pubblico-Privato (PPP) è la finanza di progetto o Project Financing (PF), ex art. 144 D.Lgs. n. 163/2006.

$\mathrm{Si}$ tratta di un istituto particolarmente versatile, spesso definito "camaleontico" in quanto adatto sia a progetti di investimenti pubblici che privati. A prescindere dalle diverse procedure attuabili, il risultato finale di un Project Financing sarà sempre la stipulazione di un contratto di concessione a favore di un veicolo societario creato ad hoc, un soggetto economico collettivo denominato Società di Progetto o Special Purpose Vehicle (SPV). Esso si occuperà interamente e unicamente della fase di realizzazione e gestione dell'operazione.

Un PF correttamente impostato, in primis in termini di programmazione di attività e costi e allocazione e trasferimento dei rischi, è capace di generare un circolo virtuoso tra spesa pubblica, attività privata e prestazione di servizi.

Il capitale privato partecipa nella realizzazione, gestione e accollo totale o parziale dei costi di opere e/o servizi pubblici o di pubblica utilità, in vista di futuri flussi di cassa operativi, garanzia primaria del rimborso del capitale investito e adeguati margini di profitto. La capacità di generare flussi di cassa che si manifestino "con adeguati livelli di certezza" è dunque passaggio fondamentale: l'opera deve essere potenzialmente redditizia.

In questo senso la fase di gestione dell'opera, saldata in modo inscindibile con quella della sua realizzazione, costituisce elemento di primaria importanza. Solo un efficiente controllo delle operazioni e un'opportuna contrattualizzazione delle obbligazioni delle parti consente di generare i flussi di cassa necessari a rimborsare il debito e remunerare gli azionisti.

In generale un'operazione di PF ha il fine di realizzare un progetto di investimento che, per essere intrapreso, si dimostri attuabile finanziariamente, tecnicamente e commercialmente; questo non solo per la durata del cantiere, 
ma per tutti i venti, trenta anni di operatività dell'opera, estendendo i calcoli economici di fattibilità.

Quando ci si pone dal punto di vista della pubblica amministrazione, la stima di convenienza dell'operazione (value for money) è determinata in misura minore dalla dimensione finanziaria del costo dell'indebitamento, e fattore discriminante diventa la realizzazione di un'opera o servizio di qualità che risponda ai fabbisogni dei cittadini.

\section{Introduzione}

Chi scrive crede che la finanza di progetto sia una valida alternativa e un'importante opportunità di dialogo tra soggetti privati e interessi pubblici responsabili, una concreta alternativa nel futuro del settore culturale, critico per emergenza e rilevanza.

Creatività e cultura fanno parte del nostro retaggio, sono ricchezza materiale e immateriale nonché carattere distintivo della nostra società, riconosciute globalmente - l'Italia vanta infatti il maggior numero di siti dichiarati patrimonio dell'umanità dall'UNESCO. Creatività e cultura hanno però anche un valore economico strategico importante, sia in termini di volume d'affari che di occupazione, ancora non sufficientemente compreso e valorizzato.

È bene specificare che il caso studio del Corpo Centrale di Villa Reale di Monza vuole essere un riferimento prima di tutto pratico, non assoluto: le numerose contestazioni sollevate per questo progetto dimostrano le debolezze, operative e strategiche, tipiche di un esperimento primo.

La restituzione di questo bene al suo pubblico rappresenta un'occasione concreta per riflettere, da molteplici prospettive, sul rapporto tra pubblico e privato, così interrelati per la prima volta. La prima, forse, di molte altre. A quasi due anni dalla riapertura della Villa proviamo a trarne conclusioni.

\section{La nostra cultura}

Considerate le condizioni di necessità in cui riversa il settore culturale italiano, le Partnership Pubblico-Privato rappresentano interessanti alternative per la Pubblica Amministrazione competente nell'ottica di adempiere ai suoi doveri costituzionali.

Un MiBACT che si trova, a causa di problematiche strutturali, a non poter disporre di un proprio portafoglio, sufficiente struttura amministrativa e adeguate capacità manageriali, rimane evidentemente limitato nelle sue attività, 
come dimostra l'esigua spesa nazionale in cultura. Positiva di certo la recente Legge di Stabilità, che prevede un incremento del bilancio del MiBACT del $27 \%$ rispetto all'anno appena trascorso e in questo senso riconosce l'inadeguatezza dei fondi messi storicamente a disposizione della cultura, e procede con un investimento necessario anche in prospettiva di futuri sviluppi.

Cresce comunque la consapevolezza dell'insufficienza della sola finanza statale tradizionale, e con essa il numero dei sostenitori dell'implementazione, anche in cultura, di forme alternative di finanziamento e management di opere e servizi pubblici. L'esperienza ha dimostrato come la partecipazione e l'integrazione del privato siano capaci di generare non solo convenienza per entrambe le parti ma anche e soprattutto beneficio per l'utenza finale, la collettività, che ottiene servizi migliori senza ulteriori aggravi fiscali e può godere nuovamente delle bellezze Italiane.

Il Project Financing si inserisce in questo quadro di convergenza tra finanziamento pubblico e privato come strumento innovativo e adeguato, ereditato dal sistema inglese e ad oggi utilizzato principalmente per la realizzazione di grandi infrastrutture o riqualificazioni di interesse pubblico. Introdotto nella regolamentazione nel 1998 e successivamente rivisitato fino alla versione ultima del 2008, è tuttavia stato impiegato in un progetto culturale solo nel 2013, in occasione della riapertura del Corpo Centrale di Villa Reale di Monza.

Come spiegare questo ritardo?

In primo luogo con una diatriba regolamentare. Nonostante sia il Regolamento di attuazione degli appalti pubblici che il Codice dei contratti pubblici prevedessero già espressamente la possibilità di utilizzo in materia culturale, la piena ammissibilità del PF nella concessione in recupero e gestione di immobili pubblici di tale interesse è stata riconosciuta solo in seguito alla dialettica processuale del caso dell'Ospedale Vecchio di Parma (2007). Assodata la liceità, rimane poi la questione in merito alla concreta praticabilità e configurabilità dell'utilizzo dello strumento, che spiegano l'ulteriore lasso di tempo trascorso.

Quando si esamina il PF si riconduce la sua prima convenienza e potenzialità alla capacità, attraverso i futuri flussi di cassa operativi, di garantire il rimborso del capitale investito e adeguati margini di profitto al finanziatore. Come e quando ciò avvenga è definito dalla regolamentazione specifica.

Nel caso dello Stato Italiano, storicamente pubblicistico e dalla tendenza a vincolare i privati in una copiosa rete di normative, la materia in merito a come la sussidiarietà verticale ed orizzontale dei progetti debba garantire la convenienza operativa ad entrambe le parti rimane contestata. Se la peculiarità di essere uno strumento regolato essenzialmente dall'autonomia privata 
ma finalizzato alla realizzazione di opere pubbliche rende la situazione particolarmente delicata, le caratteristiche intrinseche dei beni culturali aggravano ulteriormente la questione.

La natura non economicamente quantificabile e l'usuale regime di bassa redditività riducono inevitabilmente l'appeal per chi potenzialmente interessato a forme di collaborazione; qualora poi questo non scoraggiasse il privato, vi sono alte probabilità che il burocratico contesto legislativo in materia culturale ne impedisca o rallenti l'attuazione, (co)stretta tra condizioni e prescrizioni spesso sconvenienti - in questo caso un doppio regime autorizzativo.

Si tratta tuttavia solo della punta dell'iceberg.

Elemento critico primario è infatti l'esigenza posta da questi beni, che richiedono necessariamente capacità di negoziazione e convergenza collaborativa tra le parti, elementi storicamente quasi del tutto estranei all'Italia. Per ragioni storiche, culturali e sociologiche, l'intervento dei privati nella gestione del patrimonio culturale italiano non è mai stato pacificamente inteso come soluzione per una sua migliore tutela e valorizzazione.

L'Italia da sempre protegge i suoi tesori attraverso una politica culturale amministrativamente verticistica che, combinata con la (conseguente) ancestrale paura della privatizzazione della cultura, sta rallentando il processo di accettazione delle forme di partenariato.

Se l'anacronistica contrapposizione tra pubblico e privato tipicamente italiana fosse destinata a cedere, potrà farlo solo davanti all'interesse pubblico e all'inequivocabile riconoscimento del valore del patrimonio culturale. Per salvare la cultura, si deve lavorare sulla cultura.

\section{Il first mover italiano: Villa Reale di Monza}

\subsection{La Storia della Villa verso il PF}

La Villa è un complesso monumentale che risale alla seconda metà del Settecento, quando l'Imperatrice Maria Teresa d'Austria vuole costruire una residenza monumentale di campagna estiva per il figlio Ferdinando, governatore della Lombardia austriaca. Ella affida il progetto al genio dell'architetto Giuseppe Piermarini, che in soli tre anni (1777) crea un edificio monumentale in stile neoclassico, attentamente strutturato su relazioni visuali che lo legano sapientemente al territorio, per composizione ed estetica. 
La sua maestosità lo fa presto sede di governo regio, meritando l'attuale nome di Villa Reale; in questi anni si aggiunge poi il vasto Parco recintato di 750 ettari, il più grande d'Europa, ulteriore elemento di lustro.

Quest'ultimo era, inoltre, perfettamente in linea con il concetto di residenza che da sempre aveva caratterizzato il bene, concepito da promotori e realizzatori secondo una correlazione - oggi potremmo dire molto contemporanea - fra edifici e contesto, un sistema unico residenziale ma anche aperto al territorio.

Dopo la caduta di Napoleone gli Austriaci tornarono a presiederla fino alla Seconda guerra di indipendenza italiana, quando passa alla famiglia Savoia e ivi raggiunge un grandissimo splendore, adeguandosi ai mutati gusti estetici dei reali e della classe nobile. Tuttavia, dopo l'apice raggiunto con Umberto I, nel Novecento la Reggia viene di fatto abbandonata e lo scoppio della Guerra Mondiale aggrava ulteriormente la situazione facendola divenire luogo di occupazioni popolari e riparo per rifugiati, principali cause delle condizioni di urgenza ravvisate dagli anni Duemila.

Il risultato odierno è un complesso monumentale con oltre due secoli di storia e più di 700 ettari comprensivi di edifici antichi e moderni, bene pubblico proprietà di MiBACT, Regione Lombardia, Comune di Milano e Comune di Monza.

Sono dunque questi i soggetti pubblici intervenuti negli ultimi anni per risolvere lo stato di degrado e abbandono che ha depauperato la Reggia per più di un secolo, con la volontà di restituire a Monza, Milano, ma anche al Paese in generale, un bene di grande valore per la storia del territorio.

L'attenzione si è da sempre concentrata soprattutto sull'edificio monumentale più rilevante, ovvero la Villa, oggetto di ripetute ipotesi di un intervento organico purtroppo rimaste solo sulla carta, fino ad un primo tentativo risolutivo e concreto nel 2004.

In questo anno si attiva il concorso internazionale indetto dalla Regione per gli interventi sul volume architettonico della Villa e sui Giardini, con l'obiettivo di far ritrovare ad essi «una rinnovata rilevanza pubblica a carattere misto, in una convergenza di intenzionalità concordi delle diverse Istituzioni proprietarie» (Manifesto DPP del concorso, febbraio 2004).

Nei fatti il progetto di restauro vincente, portato nello specifico dal Prof. Giovanni Carbonara, non viene implementato causa le dimensioni dell'intervento. Infatti tale ambizioso progetto, per quanto metodologicamente dettagliato e "rivelativo" nella flessibilità funzionale, si presenta da subito di difficile se non impossibile realizzazione per la Pubblica Amministrazione.

Rimane però metro di giudizio e punto di riferimento condiviso per i piani di sviluppo futuri. In questo senso il progetto di Carbonara merita citazione 
nel processo che ha portato alla finalizzazione del PF: è qui che il pubblico inizia ad aprirsi e a riconoscere la doppia esigenza di recupero e restituzione del disegno, con l'introduzione di spazi nuovi dedicati a momenti di utilizzo diversi nell'ottica di un regime di autosostentamento.

\subsection{Il bando di concessione}

In data 22 marzo 2010 viene pubblicato il bando di gara sulla Gazzetta Ufficiale, redatto il 15 marzo dal Direttore Generale della procedura, Antonio Giulio Rognoni:

- Amministrazione aggiudicatrice è ILSpA, che agisce come stazione appaltante della procedura ristretta per l'affidamento di un contratto di concessione di lavori pubblici, cui oggetto specifico sono progettazione ed esecuzione della ristrutturazione del I lotto funzionale - Corpo centrale della Villa (piani nobili I e II, corpo centrale, piano terra, Belvedere, quota parte dell'Ala Nord e degli spazi esterni).

- L'importo complessivo dell'intervento è di quasi 24 milioni di euro, di cui 21 per lavori, uno per oneri di sicurezza e uno e mezzo per spese tecniche; di tale cifra è previsto che l'amministrazione aggiudicatrice corrisponda approssimativamente 19 milioni, erogati in proporzione al progressivo avanzamento dei lavori - il finanziamento residuo resta a carico del concessionario.

- Quest'ultimo avrà quale controprestazione la gestione per venti anni del corpo centrale e la parte di Ala Nord e di spazi esterni ristrutturata.

- Sono previste restrizioni di partecipazione alla gara, in merito alla situazione personale degli operatori, alla capacità tecnica e economico-finanziaria. È inoltre fissata la data massima di invio della proposta, debitamente corredata da firme e documentazioni necessarie: 7 maggio 2010, ore 12.

- Criterio generico di aggiudicazione è quello tipico dell'offerta economicamente più vantaggiosa, risultante dalla comparazione del punteggio ottenuto (max 100) attraverso la valutazione di elementi qualitativi $(60 \%)$ e quantitativi (40\%).

Si tratta, in sintesi, di un intervento di restauro e ristrutturazione del corpo centrale della Villa Reale, da "vincere" attraverso una procedura ristretta per l'affidamento della progettazione definitiva ed esecutiva e per la concessione di costruzione e gestione.

Per individuare il o i partner privati in grado di restituire la Villa ai suoi cittadini e a se stessa, il Consorzio Villa Reale e Parco di Monza opta così 
per la concessione di lavori pubblici di costruzione e gestione (o finanza di progetto ex art. 144 D.Lgs. n. 163/2006), in modo da rispondere anche alla volontà di sperimentare l'uso di strumenti innovativi nel settore dei beni culturali.

Per evitare discontinuità tra le fasi di restauro e fruizione pubblica, i comproprietari convengono di dare in concessione, oltre ai lavori di completamento della progettazione ed esecuzione del restauro, anche la successiva gestione della fruizione pubblica e dei servizi accessori connessi, così da rendere possibile la copertura dei costi della gestione continuativa.

A valle della procedura di gara indetta da ILSpA, sarebbe stato selezionato un soggetto imprenditoriale capace di garantire l'avanzamento e conclusione dei lavori di restauro (progettazione e esecuzione) e altresì provvedere alle attività di valorizzazione (gestione) rispettando quanto elaborato dal Consorzio nel Piano strategico. La scelta sarebbe dovuta ricadere insomma su un unico soggetto affidabile e articolato, con riconosciute competenze complementari e diversificate, effettivamente in grado di gestire attività sequenziali così intrinsecamente collegate.

Dopo quasi due anni dalla pubblicazione del bando, ad aggiudicarsi il progetto di restauro del Corpo Centrale o I Lotto funzionale è Italiana Costruzioni S.p.A., che costituisce - come la procedura del PF richiede - la SPV "Nuova Villa Reale di Monza S.p.A." con la minore da essa controllata, Na.Gest.

I lavori iniziano ufficialmente nel febbraio del 2012 e si concentrano su spazi che contano un totale di 10 mila $\mathrm{m} 2$ per più di 40 stanze e $3500 \mathrm{~m} 2$ di superficie. Il vasto organismo edilizio necessitava di una serie di opere di consolidamento, messa in ripristino e in sicurezza, e restauro pittorico e decorativo. Maestranze differenti hanno coabitato lavorando gomito a gomito per rispettare le scadenze programmate: più di 100 operatori tra restauratori (32), operai edili (26), meccanici (11) e altri. Il restauro si è svolto sotto la direzione di Giulia Putaturo con l'obiettivo di restituire la Villa il più possibile fedele al suo stato alla fine dell'Ottocento, rimuovendo i segni dell'incuria e riportando unità di lettura alle sale che la compongono.

Nuova Villa Reale di Monza, in quanto concessionaria della gestione degli spazi da essa stessa restaurati, ora ne dispone per due decenni in modo da poter recuperare l'investimento iniziale e dunque può determinarne l'utilizzo gestendoli direttamente o optando per una sub-concessione.

Per questo nel recupero si è dedicata alla riqualificazione degli ambienti con migliorie funzionali e tecniche che permettessero la localizzazione di nuove destinazioni d'uso e adeguamenti in linea con la fruizione e il riconoscimento della Villa come "museo di se stessa", integrando le qualità storico- 
artistiche del corpo centrale con funzionalità che andassero a sostenere il quadro economico dell'intervento.

\subsection{La Riapertura}

La Villa Reale è stata riconsegnata al suo pubblico l'8 settembre 2014. L'attuale configurazione degli spazi ha rispettato quasi perfettamente quanto previsto nel bando di concessione:

Il piano terra, dedicato all'ingresso dei visitatori e quindi alla loro accoglienza, è luogo polifunzionale, con la presenza di un bookshop, spazi ludico-formativi e, naturalmente, biglietteria e guardaroba. Sono poi state allestite le Cucine di Villa Reale, un suggestivo lounge bar arricchito da design innovativo e mosaici Liberty che ospita i ristoranti Conviviale e Gourmet, dai sapori tradizionali.

Le sale di rappresentanza del primo piano nobile, appartenute a Umberto e Margherita Savoia, hanno rispettato la vocazione legata agli eventi. La visita vera e propria è al secondo piano nobile, con gli appartamenti privati degli ospiti e dei sovrani dedicati al museo e sede di grandi mostre. Il percorso si conclude all'ultimo piano, un tempo appartamenti della servitù, con la vista sul Parco. Il Belvedere è stato progettato dall'architetto De lucchi come sede espositiva del Design Museum della Triennale di Milano (mostra "La Bellezza Quotidiana"). Seguendo il progetto del concessionario, la presenza del Triennale Design Museum prevede non solo spazi espositivi ma anche luoghi da vivere, animati da attività quali incontri, conferenza, lectures e attività didattiche.

In poco più di un semestre di attività, la Villa ha attirato circa 200 mila persone, un numero straordinario. In primo luogo i visitatori sono stati invitati a toccare con mano i risultati del restauro durante dieci giorni, dall' 8 al 18 settembre, di fruizione gratuita del complesso. Parte degli eventi di celebrazione dell'apertura e anche prima mostra offerta, "Memoria e Splendore: una storia per immagini" ha celebrato la riapertura e la restituzione della Villa al pubblico, illustrandone gli ultimi vent'anni di storia attraverso le fotografie di Piero Pozzi.

\section{Cosa si può fare}

In questa sezione si vuole rivedere l'esperienza della Villa Reale alla luce dei risultati degli ultimi due anni, traendone esempio ma anche insegnamento 
critico. La collaborazione gentilmente offerta da Attilio Navarra e dal suo gruppo ha reso l'analisi più approfondita e concreta, rafforzandone il messaggio per pubblico e privato (Exhibit 1: Draft intervista Dott. Navarra).

\subsection{La normativa vigente: $i$ rischi del promotore spontaneo}

Le difficoltà regolamentari iniziano nella definizione della gara d'appalto come singola o doppia, oppure nel definire qualora il soggetto partecipante sia anche promotore spontaneo di idee; tale elemento risulta cruciale per la conseguente rischiosità dell'iniziativa. In Italia, infatti, è possibile essere considerati a tutti gli effetti promotori di un progetto se e solo se questo viene accolto dal concessore e dichiarato di "pubblico interesse".

Questa formula, per quanto teoricamente valida, manca di effettiva applicabilità: l'azienda che decide di partecipare a una così definita gara doppia, è costretta a sopportare l'ingente (per probabilità di accadimento quanto per peso economico) rischio di non ottenere il consenso e la dichiarazione necessaria. Il risultato è che l'investimento in ricerca e sviluppo - già fatto rimarrà a carico solo suo.

È bene sottolineare che tale scelta di metodo è motivata dal voler promuovere l'iniziativa e la creatività dei soggetti partecipanti, che godono così di maggiore libertà: possono proporre, prima, e realizzare, poi, un progetto da loro studiato. Tuttavia, potersi permettere di perdere tanto tempo e tante energie, mentali ed economiche, è indiscutibilmente possibilità propria solo di realtà di determinate dimensioni. Ne consegue che il risultato finale non è più un valore aggiunto nuovo e esterno, ma piuttosto una limitazione delle aziende partecipanti e proponenti.

Le suddette riflessioni si possono riportare nel caso del Corpo Centrale della Villa Reale di Monza, dove la definizione della gara come singola ha certamente stimolato la partecipazione di piccole e medie realtà: 14 gruppi si contendevano l'aggiudicazione. Il secondo elemento a destare interesse è stato poi la forte presenza del Pubblico in investimenti e contributi, pronto a finanziare più del $70 \%$ del progetto.

Questi due vantaggi, saggiamente combinati dal concedente, Infrastrutture Lombarde S.p.A., hanno reso questa gara un uniquum. Ciò che ha allontanato molti dei proponenti in un secondo momento è stata indubbiamente la dimensione del piano economico finanziario richiesto, che si andava sommando al rischio di non poter avere un precedente, un caso concreto a cui fare riferimento. Sono così rimasti a formalizzare l'offerta e contendersi l'aggiudicazione solo Italiana Costruzioni e SCAI. 


\subsection{Ideologia: una possibile soluzione}

Una politica culturale amministrativamente verticistica e un'ancestrale paura della privatizzazione della cultura hanno sicuramente rallentato il possibile miglioramento del rendimento e della fruizione del patrimonio culturale attraverso forme di partenariato.

Dagli anni Novanta si sono registrate alcune aperture, come l'approvazione della legge Ronchey (1993), a cui però è seguito un percorso di alti e bassi, costellato di dibattiti e intoppi motivati da pregiudizio e ritrosia nel vedere lo Stato trasformarsi da gestore a regolatore. Dimostrazione di ciò sta anche nello stesso Codice dei Beni Culturali del 2004, la cui impostazione di fondo rivela un legislatore prevalentemente "conservativo" che pone la valorizzazione del patrimonio in una posizione residuale rispetto a tutela e conservazione (art.6, c. 2: «in forme compatibili con la tutela e tali da non pregiudicarne le esigenze»); nelle attività di valorizzazione, poi, i privati for profit hanno potuto rivestire un ruolo solo secondario rispetto agli interventi pubblici.

L'emergente esigenza di contenimento della spesa pubblica imposta alle pubbliche amministrazioni e la copiosità degli interventi necessari, da un lato, e la possibilità che il coinvolgimento dei privati costituisca un rilancio della cultura italiana come motore artistico e economico, dall'altro, hanno progressivamente lottato contro un conservatorismo tipicamente nostrano. Le circostanze di necessità inducono molti ad auspicare un apporto e convoglio di capitali privati sulla base di motivazioni anche diverse dalla sola "arte per l'arte" - ovvero in forma di mecenatismo, di interesse puro per l'arte avulso dall'idea di profitto.

Si cerca di parlare sempre di più di imprenditoria culturale, perché discuterne e promuoverne le prospettive è il passo iniziale per dare nuovi spunti e nuova forza alle PPP e iniziative simili a quanto avvenuto a Monza. Ė positivo notare come le proposte in questa direzione provengano tanto dal pubblico come dal privato.

L'introduzione degli incentivi fiscali noti complessivamente come "ArtBonus" (Decreto per la cultura n. 83/2014) è parte di questo movimento e ha l'obiettivo di incentivare i contributi privati nella manutenzione e nel restauro di beni culturali pubblici, nonché realizzazione di nuove strutture. $\mathrm{Si}$ tratta di agevolazioni fiscali applicate ad erogazioni liberali in denaro a sostegno della cultura e dello spettacolo, che consentono di detrarre in 3 anni il 65\% dell'importo erogato nel 2015 (50\% dal 2016). Questa iniziativa ha portato, tra le altre, la manutenzione dell'Arena di Verona grazie al contributo di Unicredit o la Basilica della Santissima Annunziata (Firenze) con l'intervento del Gruppo Ferragamo. 
Anche i privati, consapevoli della delicata situazione, si propongono come possibili interlocutori tra finanziatori e Amministrazione, facendo leva su strumenti diversi. Si può portare ad esempio la recente iniziativa di LoveItaly!, associazione senza fini di lucro creata dalla holding LVenture Group e rappresentata dal Presidente Prof. Richard Hodges. L'idea è quella di tutelare, promuovere e valorizzare il patrimonio culturale Italiano partecipando al sostegno dei progetti di restauro attraverso piattaforme crowdfounding. L'attività di LoveItaly! nasce da un amore sincero per le bellezze artistiche Italiane e dalla consapevolezza che questo sia condiviso ben oltre $\mathrm{i}$ nostri confini territoriali, così che si possa riunire gli sforzi e i desideri di milioni di appassionati della storia e della cultura italiana in generosi e fruttuosi contributi.

\subsection{Imprenditorialità: la questione "sostenibilità"}

Spesso si additano i beni culturali di non poter rientrare nella categoria di interventi realizzabili attraverso la finanza di progetto richiamando il loro essere "opere fredde", contrario alla logica di produzione di futuri cash flows che contraddistingue questo strumento finanziario.

Per trattare tale questione si sceglie di far riferimento e sostenere quanto evidenziato nel ciclo di convegni istituzionali organizzati dai fratelli Attilio e Luca Navarra, titolari di Italiana Custruzioni S.p.A., nonché gestori del Corpo Centrale di Villa Reale. Si tratta di una serie di incontri in luoghi culturali di alto rilievo (Venaria Reale, Matera, Venezia, L'Aquila, Monza e conclusione ad Istanbul) nel corso del biennio 2015-2017, in cui grandi esponenti della cultura italiana, imprenditoria e istituzioni si confrontano in tavole rotonde su temi di PPP.

Tale iniziativa, a cura di Francesco Maggiore, con il coordinamento scientifico di Francesco Moschini, prende spunto dal progetto "Valore Restauro Sostenibile dei Fratelli Navarra". Quest'ultimo propone una "Italian way of doing restoration", un restauro made in Italy che nasce dalle esperienze acquisite e consolidate in decenni di grandi progetti, e si rinnova come laboratorio sperimentale per dare nuova definizione al restauro.

La parola chiave diventa Sostenibilità. In primis di certo sostenibilità economico-finanziaria, ma anche sociale e ambientale. Questi luoghi non sono solo luoghi di rilievo, ma di ritrovo. Riprendendo l'estratto per la presentazione del progetto di Italiana Costruzioni, possiamo definire le diverse sfaccettature di sostenibilità come: 
- ambientale: privilegiare il recupero alla nuova edificazione, per tutelare i paesaggi e il territorio, e utilizzare risorse e energie in modo quanto più sostenibile, grazie al ricorso alle nuove tecnologie;

- economica: la governance si deve impegnare nel creare sinergie economiche e finanziarie, in particolare nei rapporti tra pubblico e privato, verso una migliore salvaguardia e valorizzazione dell'oggetto di intervento;

- sociale: un impegno nella promozione di attività ausiliare di formazione al lavoro, educazione civile e coinvolgimento comunitario dei cittadini.

La responsabilità della conservazione del valore dei beni monumentali e del loro contesto si basa su una governance consapevole e capace di sintetizzare molteplici discipline complementari e educare alla manutenzione programmata e preventiva. Come evidenziato in precedenza, quello italiano è il più consistente patrimonio artistico e culturale al mondo e dunque richiede competenze e risorse commisurate.

Quest'ultimo elemento è particolarmente caro a Attilio Navarra, che sottolinea la trasmissione dei valori culturali alle future generazioni come principale dovere nel lavoro suo e di Italiana Costruzioni S.p.A. Purtroppo, la tipica organizzazione Statale caratterizzata da interventi e finanziamenti a pioggia, non strutturati, mostra solo oggi i suoi esiti negativi e "crolla" insieme alle mura e i tetti dei monumenti. Occorre mettere il passato al servizio del futuro, riservando a entrambi la stessa cura.

Diventa dunque importante promuovere un'attenzione maggiore alle tecniche di salvaguardia del patrimonio culturale e artistico italiano, e in questo processo l'indotto imprenditoriale dei privati, italiani e non, può rappresentare un'opportunità in più.

\section{Riferimenti bibliografici}

Atto di Costituzione "Consorzio Villa Reale e Parco di Monza”, n. 3109 Repertorio, n. 1328 Raccolta, 20 luglio 2009.

Azzolini, V., "Fare cultura è un'impresa", le sponsorizzazioni culturali in Italia, IBL Special Report, 24 febbraio 2015.

Carbonara, G., Progetto preliminare di Recupero e valorizzazione della Villa Reale di Monza e dei Giardini di pertinenza - Stralcio della Relazione Illustrativa, 2004.

Carpentieri, P., La gestione dei beni culturali e la finanza di progetto, in Finanza di progetto. Temi e prospettive. Approfondimenti sistematici ed interdisciplinari, a cura di G.F. Cartei, M. Ricchi, Napoli, 2010. 
Cederna, A., Comitato per il Parco di Monza, "Il restauro della Villa Reale di Monza: chi l'ha pagato e chi ci guadagna - un restauro spinoso" (Monza, 2014, 26 ottobre). -- Tratto da Brianza Popolare: http://www.brianzapopolare.it/sezioni/cultura/index.php.

Consiglio di Stato, sez. IV, 11 luglio 2008, n. 3507.

Consiglio di Stato, sez. IV, 23 luglio 2009, n. 4639.

Consorzio Villa Reale e Parco di Monza, "Il Consorzio Villa Reale e Parco di Monza cerca sponsor”, Comunicato Stampa n. 5, Monza, 19 febbraio 2015.

Consorzio Villa Reale e Parco di Monza, "La Villa Reale di Monza si può salvare. Ecco cosa stiamo facendo", -- http://www.reggiadimonza.it.

Crippa, M.A., Antecedenti procedurali e di progetto al recupero funzionale e al restauro del corpo centrale della Villa Reale di Monza.

D.Lgs. 22 gennaio 2004, n. 42 (e successive integrazioni), Codice dei Beni Culturali e del Paesaggio.

D.Lgs. n. 163/2006, Codice dei contratti pubblici, Capo II Titolo IV Parte II.

D.P.R. 5 ottobre 2010, n. 207, Regolamento di attuazione degli appalti pubblici (Titolo XI, Lavori riguardanti i beni del patrimonio culturale).

Ernst \& Young Financial-Business Advisors S.p.A., "Italia Creativa. Primo studio sull'Industria della Cultura e della Creatività in Italia", 18 gennaio 2016 -(http://www.italiacreativa.eu/).

Giovannini, R., "Project Financing e confronto con gli altri strumenti di PPP - Partenariato pubblico privato per i servizi di gestione, valorizzazione e riqualificazione energetica del patrimonio dei comuni”, XIX Assemblea Annuale ANCI, 19 ottobre 2012.

http://www.reggiadimonza.it/RESTAURO_VILLA_REALE/270.

Il Giorno, "Villa Reale pronta per Expo: restauro finito, apertura a settembre" (2014, giugno 26). -- Tratto da Monza Brianza: http://www.ilgiorno.it/monza/cronaca/ 2014/06/26/1083585-villa-reale.shtml.

Infrastrutture Lombarde S.p.A, "Lavori di ristrutturazione-2010/S 53-078354. Concessione di lavori pubblici", 15 marzo 2010 (pubblicazione G.U. 22 marzo).

Infrastrutture Lombarde S.p.A, "Recupero e valorizzazione della villa reale di Monza e dei giardini di pertinenza. I lotto funzionale - Corpo centrale. Struttura della Concessione".

Infrastrutture Lombarde S.p.A, "Recupero e valorizzazione della villa reale di Monza e dei giardini di pertinenza. I lotto funzionale - Corpo centrale. Le funzioni insediate".

Italiana Costruzioni S.p.A., "Progetto Valore Restauro Sostenibile dei Fratelli Navarra".

Lev Mannheimer, G., "Quando valorizzare costa poco o nulla (ai contribuenti). Il project financing nei beni culturali”, IBL Briefing Paper n. 145, 24 febbraio 2015.

Michiara, P., La finanza di progetto nei beni culturali, in «Aedon - Rivista di arti e diritto online», I, 2008 -- (www.aedon.mulino.it).

Ministero per i Beni e le Attività Culturali, Regione Lombardia, Comune di Monza e Comune di Milano, Accordo Strategico, 30 luglio 2008.

Sentenze TAR Emilia Romagna, Parma, sez. I, 4 dicembre 2007, n. 618; sez. I, 3 giugno 2008, n. 304. 